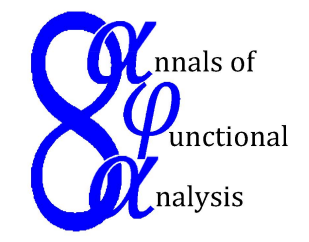

Ann. Funct. Anal. 5 (2014), no. 2, 61-73

$\mathscr{A}$ NNALS OF $\mathscr{F}$ UNCTIONAL $\mathscr{A}$ NALYSIS

ISSN: 2008-8752 (electronic)

URL:www.emis.de/journals/AFA/

\title{
TIME SCALES HARDY-TYPE INEQUALITIES VIA SUPERQUADRACITY
}

\author{
JAMES ADEDAYO OGUNTUASE ${ }^{1} *$ AND LARS-ERIK PERSSON $^{2}$ \\ This paper is dedicated to Professor Tsuyoshi Ando in celebration of his distinguished \\ achievements in Matrix Analysis and Operator Theory \\ Communicated by V. Valov
}

\begin{abstract}
In this paper some new Hardy-type inequalities on time scales are derived and proved using the concept of superquadratic functions. Also, we extend Hardy-type inequalities involving superquadratic functions with general kernels to the case with arbitrary time scales. Several consequences of our results are given and their connection with recent results in the literature are pointed out and discussed.
\end{abstract}

\section{INTRODUCTION}

In a note published in 1920, Hardy [7] (see also [9]) announced (without proof) that if $p>1$ and $f \geq 0$ is a $p$-integrable function over the finite interval $(0, x)$, then

$$
\int_{0}^{\infty}\left(\frac{1}{x} \int_{0}^{x} f(t) d t\right)^{p} d x \leq\left(\frac{p}{p-1}\right)^{p} \int_{0}^{\infty} f^{p}(x) d x
$$

holds and the constant $\left(\frac{p}{p-1}\right)^{p}$ is the best possible. Inequality (1.1) which is usually referred to in the literature as the classical Hardy inequality, was proved in 1925 by Hardy [8] (see also [9, 10, 11, 12, 13] for the prehistory, history, and further development of this inequality). In a recent paper, Oguntuase and Persson [14] gave a review of recent development of Hardy inequality using the convexity argument and the concept of superquadracity introduced by Abramovich et al.

Date: Received: October 28, 2013; Accepted: November 2, 2013.

* Corresponding author.

2010 Mathematics Subject Classification. Primary 46G12; Secondary 47B34; 47J20.

Key words and phrases. Time scales, Hardy-type inequalities, superquadratic functions, subquadratic function, Hardy-Knopp type inequalities. 
[1] while Donchev et al. [6] obtained some Hardy-type inequalities with general kernels on time scales for multivariate convex functions.

Stefan Hilger (see e.g. [4, 5]) initiated the calculus of time scales in order to create a theory that can unify discrete and continuous analysis. This new concept has inspired researchers to study Hardy inequality on time scales. The history of the Hardy inequality on time scales seems to have been initiated by Řehák [19] in an attempt to unify and extend the classical Hardy integral inequality and the discrete Hardy inequality by means of the theory of time scales.

First we recall some basic concepts used in this paper and also refer interested reader to the books $[4,5]$ for a detailed theory of time scales. A time scale is an arbitrary nonempty closed subset of the real numbers $\mathbb{R}$.

Definition 1.1. Let $\mathbb{T}$ be a time scale. For $t \in \mathbb{T}$, we define the forward jump operator $\sigma: \mathbb{T} \rightarrow \mathbb{T}$ by

$$
\sigma(t)=\inf \{s \in \mathbb{T}: s>t\} \quad \text { for all } \quad t \in \mathbb{T},
$$

while the backward jump operator $\rho: \mathbb{T} \rightarrow \mathbb{T}$ is defined by

$$
\rho(t)=\sup \{s \in \mathbb{T}: s<t\} \quad \text { for all } \quad t \in \mathbb{T} .
$$

The point $t$ is said to be right-scattered if $\sigma(t)>t$, respectively left-scatted if $\rho(t)<t$. Points that are right-scattered and left-scattered at the same time are called isolated. The point $t$ is called right-dense if $t<\sup \mathbb{T}$ and $\sigma(t)=t$, respectively left-dense if $t>\inf \mathbb{T}$ and $\rho(t)=t$. Finally, the graininess function $\mu: \mathbb{T} \rightarrow[0, \infty)$ is defined by

$$
\mu(t)=\sigma(t)-t \quad \text { for all } \quad t \in T .
$$

A mapping $f: \mathbb{T} \rightarrow \mathbb{R}$ is said to be rd-continuous if

(i) $f$ is continuous at each right-dense point or maximal point of $\mathbb{T}$;

(ii) at each left-dense point $t \in \mathbb{T}, \lim _{s \rightarrow t^{-}} g(s)=g\left(t^{-}\right)$exists.

The set of all rd-continuous functions from $\mathbb{T} \rightarrow \mathbb{R}$ is usually denoted by $C_{r d}(\mathbb{T}, \mathbb{R})$.

Let $\mathbb{T}$ be a time scale and $[a, b) \subset \mathbb{R}$. The Lebesgue integral associated with the measure $\mu$ on $[a, b)$ is called the Lebesgue $\Delta$-integral. If $f:[a, b) \rightarrow \mathbb{R}$, the corresponding $\Delta$-integral of $f$ over $[a, b)$ will be denoted by $\int_{a}^{b} f(t) \Delta t$. If $\mathbb{T}$ is a time scale and the interval $[a, b) \subset \mathbb{T}$ consists of isolated points, then

$$
\int_{a}^{b} f(t) \Delta t=\sum_{t \in[a, b)}(\sigma(t)-t) f(t)
$$

In 2005, ̌̌ehák [19] stated that if $a>0, p>1$, and $f$ be a nonnegative function such that the delta integral $\int_{a}^{\infty}(f(s))^{p} \Delta s$ exists as a finite number, then

$$
\int_{a}^{\infty}\left(\frac{1}{\sigma(x)-a} \int_{a}^{\sigma(x)} f(t) \Delta t\right)^{p} \Delta x<\left(\frac{p}{p-1}\right)^{p} \int_{a}^{\infty} f^{p}(x) \Delta x
$$

unless $f \equiv 0$. If, in addition, $\mu(t) / t \rightarrow 0$ as $t \rightarrow \infty$, then the constant $\left(\frac{p}{p-1}\right)^{p}$ is the best possible. 
In 2008, Özakan and Yildirim [17] gave a time scale Hardy inequality involving several functions and also derived a time scale Hardy-Knopp type inequality. Furthermore, the same authors in another paper [18] further obtained a generalization of Hardy-Knopp's type inequality for several functions and also derived the Hardy-Knopp type inequality with a general kernel.

We also need the following definition of superquadratic functions:

Definition 1.2. (See [1, Definition 2.1]). A function $\phi:[0, \infty)$ is called superquadratic provided that for all $x \geq 0$ there exists a constant $C_{x} \in \mathbb{R}$ such that

$$
\phi(y)-\phi(x)-\phi(|y-x|) \geq C_{x}(y-x)
$$

for all $y \geq 0$.

We say that $\phi$ is subquadratic if $-\phi$ is superquadratic.

A number of Hardy-type inequalities have recently been derived by using a powerful convexity technique, see [14] and the references there. By instead of convex functions using superquadratic functions some similar refined Hardy-type inequalities can be derived. The first result in this direction can be found in [15] (see also [16]) for the multidimensional analogue.

Our aim in this paper is to further develop and unify these techniques and derive, prove and discuss some new refined Hardy-type inequalities on time scales using the concept of superquadracity. Furthermore, we extend Hardy-type inequalities involving superquadratic functions with general kernels to arbitrary time scales.

The results in Section 2 may be regarded as a generalization to this time scale setting of the new refined Hardy-type inequalities with "breaking point" $p=2$ recently published in [15]. In Section 3 we prove the corresponding result for the more general case when the Hardy operator is replaced by a Hardy type operator with general positive kernel. Moreover, these results are given even in a multidimensional setting.

\section{SOME TIME SCALE INEQUALITIES FOR SUPERQUADRACITY}

In a recent paper Bibi et al.[3] established the Fubini's theorem on time scales while Barić et al.[2] obtained a refined Jensen's inequality on time scales for superquadratic functions. These results are very useful in the proofs of our main results and for the reader's convenience, we recall these results before stating our main results in this section.

Lemma 2.1. [3, Theorem 1.1] (Fubini' theorem on Time Scales). Let $\left(\Omega, \mathcal{M}, \mu_{\Delta}\right)$ and $\left(\Lambda, \mathcal{L}, \lambda_{\Delta}\right)$ be two finite dimensional time scale measures spaces. If $f$ : $\Omega \times \Lambda \rightarrow \mathbb{R}$ is a $\mu_{\Delta} \times \lambda_{\Delta}$-integrable functions and define the function $\phi(y)=$ $\int_{\Omega} f(x, y) \Delta x$ for a.e. $y \in \Lambda$ and $\psi(x)=\int_{\Lambda} f(x, y) \Delta y$ for a.e. $x \in \Omega$, then $\phi$ is $\lambda_{\Delta}$-integrable on $\Lambda, \psi$ is $\mu_{\Delta}$-integrable on $\Omega$ and

$$
\int_{\Omega} \Delta x \int_{\Lambda} f(x, y) \Delta y=\int_{\Lambda} \Delta y \int_{\Omega} f(x, y) \Delta x .
$$


Lemma 2.2. (See $\left[2\right.$, Theorem 2.5]). Let $a, b \in \mathbb{T}$. Suppose $f:[a, b]_{\mathbb{T}^{k}} \rightarrow[0, \infty)$ is rd-continuous and $\phi:[0, \infty) \rightarrow \mathbb{R}$ is continuous and superquadratic. Then

$\phi\left(\frac{1}{b-a} \int_{a}^{b} f(t) \Delta t\right) \leq \frac{1}{b-a} \int_{a}^{b}\left[\phi(f(s))-\phi\left(\left|f(s)-\frac{1}{b-a} \int_{a}^{b} f(t) \Delta t\right|\right)\right] \Delta s$.

Now we state and prove a strengthened form of a refined Hardy-type inequality for superquadratic functions.

Theorem 2.3. Let $u \in C_{r d}([a, b], \mathbb{R})$ be a nonnegative function such that the $\Delta$-integral $\int_{t}^{b} \frac{u(t)}{(x-a)(\sigma(x)-a)} \Delta x<\infty$ and define the weight function $v$ by

$$
v(t)=(t-a) \int_{t}^{b} \frac{u(x)}{(x-a)(\sigma(x)-a)} \Delta x, \quad t \in(a, b) .
$$

(a) If the real-valued function $\phi$ is superquadratic on $(a, b), 0<a<b \leq \infty$, then

$$
\begin{aligned}
& \int_{a}^{b} u(x) \phi\left(\frac{1}{\sigma(x)-a} \int_{a}^{\sigma(x)} f(t) \Delta t\right) \frac{\Delta x}{x-a} \\
& +\int_{a}^{b} \int_{t}^{b} \phi\left(\left|f(t)-\frac{1}{\sigma(x)-a} \int_{a}^{\sigma(x)} f(t) \Delta t\right|\right) \frac{u(x)}{(x-a)(\sigma(x)-a)} \Delta x \Delta t \\
& \leq \int_{a}^{b} v(x) \phi(f(x)) \frac{\Delta x}{x-a}
\end{aligned}
$$

holds for all $\Delta$-integrable function $f \in C_{r d}([a, b], \mathbb{R})$ such that $f(x) \in(a, c)$.

(b) If the real-valued function $\phi$ is subquadratic on $(a, c), 0<a<c \leq \infty$, then (2.3) holds in the reversed direction.

Proof. (a) By using the refined Jensen inequality (2.2) and the Fubini theorem (2.1) on time scales to the first term on the left hand side of (2.3), we find that

$$
\begin{aligned}
& \int_{a}^{b} u(x) \phi\left(\frac{1}{\sigma(x)-a} \int_{a}^{\sigma(x)} f(t) \Delta t\right) \frac{\Delta x}{x-a} \\
\leq & \int_{a}^{b} \frac{u(x)}{(x-a)(\sigma(x)-a)} \int_{a}^{\sigma(x)} \phi(f(t)) \Delta t \Delta x \\
& -\int_{a}^{b} \frac{u(x)}{(x-a)(\sigma(x)-a)} \int_{t}^{b} \phi\left(\left|f(t)-\frac{1}{\sigma(x)-a} \int_{a}^{\sigma(x)} f(t) \Delta t\right|\right) \Delta t \Delta x \\
= & \int_{a}^{b} \phi(f(t)) \int_{t}^{b} \frac{u(x)}{(x-a)(\sigma(x)-a)} \Delta x \Delta t \\
& -\int_{a}^{b} \int_{t}^{b} \phi\left(\left|f(t)-\frac{1}{\sigma(x)-a} \int_{a}^{\sigma(x)} f(t) \Delta t\right|\right) \frac{u(x)}{(x-a)(\sigma(x)-a)} \Delta x \Delta t
\end{aligned}
$$




$$
\begin{aligned}
= & \int_{a}^{b} v(t) \phi(f(t)) \frac{\Delta t}{(t-a)} \\
& -\int_{a}^{b} \int_{t}^{b} \phi\left(\left|f(t)-\frac{1}{\sigma(x)-a} \int_{a}^{\sigma(x)} f(t) \Delta t\right|\right) \frac{u(x)}{(x-a)(\sigma(x)-a)} \Delta x \Delta t .
\end{aligned}
$$

(b) This is similar to the proof of (a) above but the only difference is that in this case the inequality sign is reversed. The proof is complete.

We now consider Theorem 2.3 in some special cases. First we note that if we set $u(x) \equiv 1$, then we obtain that

$$
v(x)=\left\{\begin{array}{cc}
1-\frac{x-a}{b-a} & \text { if } b<\infty \\
1 & \text { if } b=\infty .
\end{array}\right.
$$

Hence, we have the following:

Corollary 2.4. Let the assumptions of Theorem 2.3 be satified.

(a) If $\phi$ is superquadratic and $a<b<\infty$, then

$$
\begin{aligned}
& \int_{a}^{b} \phi\left(\frac{1}{\sigma(x)-a} \int_{a}^{\sigma(x)} f(t) \Delta t\right) \frac{\Delta x}{x-a} \\
& +\int_{a}^{b} \int_{t}^{b} \phi\left(\left|f(t)-\frac{1}{\sigma(x)-a} \int_{a}^{\sigma(x)} f(t) \Delta t\right|\right) \frac{\Delta x}{(x-a)(\sigma(x)-a)} \Delta t \\
& \leq \int_{a}^{b}\left(1-\frac{x-a}{b-a}\right) \phi(f(x)) \frac{\Delta x}{x-a},
\end{aligned}
$$

and

$$
\begin{aligned}
& \int_{a}^{\infty} \phi\left(\frac{1}{\sigma(x)-a} \int_{a}^{\sigma(x)} f(t) \Delta t\right) \frac{\Delta x}{x-a} \\
& +\int_{a}^{\infty} \int_{t}^{\infty} \phi\left(\left|f(t)-\frac{1}{\sigma(x)-a} \int_{a}^{\sigma(x)} f(t) \Delta t\right|\right) \frac{\Delta x}{(x-a)(\sigma(x)-a)} \Delta t \\
& \leq \int_{a}^{\infty} \phi(f(x)) \frac{\Delta x}{x-a} .
\end{aligned}
$$

(b) The inequalities (2.4) and (2.5) hold in the reversed direction if $\phi$ is subquadratic.

Example 2.5. By taking $\mathbb{T}=\mathbb{R}$ and $a=0$ in Corollary 2.4, inequalities (2.4) and (2.5) read:

$$
\begin{aligned}
& \int_{0}^{b} \phi\left(\frac{1}{x} \int_{0}^{x} f(t) d t\right) \frac{d x}{x}+\int_{0}^{b} \int_{t}^{b} \phi\left(\left|f(t)-\frac{1}{x} \int_{0}^{x} f(t) d t\right|\right) \frac{d x}{x^{2}} d t \\
& \leq \int_{0}^{b}\left(1-\frac{x}{b}\right) \phi(f(x)) \frac{d x}{x}
\end{aligned}
$$


and

$$
\begin{aligned}
& \int_{0}^{\infty} \phi\left(\frac{1}{x} \int_{0}^{x} f(t) d t\right) \frac{d x}{x}+\int_{0}^{\infty} \int_{t}^{\infty} \phi\left(\left|f(t)-\frac{1}{x} \int_{0}^{x} f(t) d t\right|\right) \frac{d x}{x^{2}} d t \\
& \leq \int_{0}^{\infty} \phi(f(x)) \frac{d x}{x},
\end{aligned}
$$

respectively.

Remark 2.6. The inequalities (2.6) and (2.7) coincide with Proposition 2.1 from [15], written for the case $\mathrm{u}(\mathrm{x})=1$.

By using the well-known fact that the function $\phi(u)=u^{p}$ is superquadratic for $p \geq 2$ and subquadratic for $1<p \leq 2$ we obtain the following:

Example 2.7. Assume that $\phi(u)=u^{p}$ and $\int_{a}^{b} f^{p}(x) \frac{\Delta x}{x-a}<\infty$. Then inequalities (2.4) and (2.5) read:

$$
\begin{aligned}
& \int_{a}^{b}\left(\frac{1}{\sigma(x)-a} \int_{a}^{\sigma(x)} f(t) \Delta t\right)^{p} \frac{\Delta x}{x-a} \\
& +\int_{a}^{b} \int_{t}^{b}\left|f(t)-\frac{1}{\sigma(x)-a} \int_{a}^{\sigma(x)} f(t) \Delta t\right|^{p} \frac{\Delta x}{(x-a)(\sigma(x)-a)} \Delta t \\
& \leq \int_{a}^{b}\left(1-\frac{x-a}{b-a}\right) f^{p}(x) \frac{\Delta x}{x-a}
\end{aligned}
$$

for $a<b<\infty$ and

$$
\begin{aligned}
& \int_{a}^{\infty}\left(\frac{1}{\sigma(x)-a} \int_{a}^{\sigma(x)} f(t) \Delta t\right)^{p} \frac{\Delta x}{x-a} \\
& +\int_{a}^{\infty} \int_{t}^{\infty}\left|f(t)-\frac{1}{\sigma(x)-a} \int_{a}^{\sigma(x)} f(t) \Delta t\right|^{p} \frac{\Delta x}{(x-a)(\sigma(x)-a)} \Delta t \\
& \leq \int_{a}^{\infty} f^{p}(x) \frac{\Delta x}{x-a}
\end{aligned}
$$

respectively. Moreover, if $1<p \leq 2$, then the inequalities (2.8) and (2.9) hold in the reversed direction.

Remark 2.8. By taking $\mathbb{T}=\mathbb{R}$ and $a=0$ in Example 2.7, inequality (2.8) coincides with inequality (3.2) in [15].

Remark 2.9. The natural "breaking point" (the point where the inequality reverses) in Hardy type inequalities is usually $p=1$. However, here we see that for our refined Hardy type inequality the natural breaking point is $p=2$ and even more remarkable for $p=2$ we have a new identity even in our case with time scales. 


\section{REFINED TIME SCALES HARDY-TYPE INEQUALITIES FOR SUPERQUADRATIC FUNCTIONS AND HARDY OPERATORS WITH GENERAL KERNELS}

Our first main result in this Section reads:

Theorem 3.1. Let $\left(\Omega_{1}, \Sigma_{1}, \mu_{\Delta_{1}}\right)$ and $\left(\Omega_{2}, \Sigma_{1}, \mu_{\Delta_{2}}\right)$ be two time scale measure spaces with positive $\sigma-$ finite measures and let $u: \Omega_{1} \rightarrow \mathbb{R}$ and $k: \Omega_{1} \times \Omega_{2} \rightarrow \mathbb{R}$ be nonnegative such that $k(x,$.$) is a \mu_{\Delta_{2}}$-integrable function for $x \in \Omega_{1}$. Furthermore, suppose that $K: \Omega_{1} \rightarrow \mathbb{R}$ is defined by

$$
K(x):=\int_{\Omega_{2}} k(x, y) \Delta \mu_{2}(y)>0 . \quad x \in \Omega_{1},
$$

and

$$
v(y):=\int_{\Omega_{2}} u(x) \frac{k(x, y)}{K(x)} \Delta \mu_{1}(x)<\infty, \quad y \in \Omega_{2} .
$$

If $\phi:[a, \infty) \rightarrow \mathbb{R}(a \geq 0)$ is a nonnegative superquadratic function, then the inequality

$$
\begin{aligned}
& \int_{\Omega_{2}} u(x) \phi\left(A_{k} f(x)\right) \Delta \mu_{1}(x) \\
& +\int_{\Omega_{2}} \int_{\Omega_{2}} u(x) \frac{k(x, y)}{K(x)} \phi\left(\left|f(y)-A_{k} f(x)\right|\right) \Delta \mu_{1}(x) \Delta \mu_{2}(y) \\
& \leq \int_{\Omega_{2}} v(x) \phi(f(x)) \Delta \mu_{2}(x)
\end{aligned}
$$

holds for all nonnegative $\mu_{\Delta_{2}}$-integrable function $f: \Omega_{2} \rightarrow \mathbb{R}$ and for $A_{k} f:$ $\Omega_{1} \rightarrow \mathbb{R}$ defined by

$$
A_{k} f(x)=\frac{1}{K(x)} \int_{\Omega_{2}} k(x, y) f(y) \Delta \mu_{2}(y), \quad x \in \Omega_{1} .
$$

If $\phi$ is subquadratic, then the inequality sign in (3.1) is reversed.

Proof. The proof follows by using Jensen's inequality (2.2) and the Fubini theorem (2.1) on time scales. Indeed, we obtain that 


$$
\begin{aligned}
& \int_{\Omega_{2}} u(x) \phi\left(A_{k} f(x)\right) \Delta \mu_{1}(x) \\
= & \int_{\Omega_{2}} u(x) \phi\left(\frac{1}{K(x)} \int_{\Omega_{2}} k(x, y) f(y) \Delta \mu_{2}(y)\right) \Delta \mu_{1}(x) \\
\leq & \int_{\Omega_{1}} \frac{u(x)}{K(x)}\left(\int_{\Omega_{2}} k(x, y) \phi(f(y)) \Delta \mu_{2}(y)\right) \Delta \mu_{1}(x) \\
& -\int_{\Omega_{1}} \frac{u(x)}{K(x)} \int_{\Omega_{2}} k(x, y) \phi\left(\left|f(y)-A_{k} f(x)\right|\right) \Delta \mu_{2}(y) \Delta \mu_{1}(x) \\
= & \int_{\Omega_{2}} \phi(f(y))\left(\int_{\Omega_{1}} \frac{u(x) k(x, y)}{K(x)} \Delta \mu_{1}(x)\right) \Delta \mu_{2}(y) \\
& -\int_{\Omega_{2}} \int_{\Omega_{1}} \frac{u(x) k(x, y)}{K(x)} \phi\left(\left|f(y)-A_{k} f(x)\right|\right) \Delta \mu_{1}(x) \Delta \mu_{2}(y)
\end{aligned}
$$

from which (3.1) follows.

The proof of the case in which $\phi$ is subquadratic is similar the only difference is that the inequality sign in (3.1) is reversed. The proof is complete.

Example 3.2. In Theorem 3.1, let $\Omega_{1}=\Omega_{2}=[a, b)_{\mathbb{T}}, 0 \leq a<b \leq \infty$, where $\mathbb{T}$ is a time scale, replace the time scale measures $\Delta \mu_{1}(x)$ and $\Delta \mu_{2}(y)$ by the Lebesgue scale measures $\Delta x$ and $\Delta y$ respectively, and also replace $u(x), v(y)$ and $k(x, y)$ by $\frac{u(x)}{x-a}, \frac{w(y)}{y-a}$ and $k(x, y)=1$, if $0 \leq y \leq \sigma(x), k(x, y)=0$ if $y>\sigma(x)$, respectively. In this setting we have

$$
K(x)=\int_{a}^{\sigma(x)} k(x, t) \Delta t=\sigma(x)-a>0 . \quad x \in[a, b)_{\mathbb{T}},
$$

and

If we let

$$
A_{k} f(x)=\frac{1}{(\sigma(x)-a)} \int_{a}^{\sigma(x)} f(t) \Delta t, \quad x \in \Omega_{1} .
$$

$$
v(t)=(t-a) \int_{t}^{b} u(x) \frac{\Delta x}{(\sigma(x)-a)(x-a)}<\infty, \quad t \in[a, b)_{\mathbb{T}},
$$

then we obtain inequality

$$
\begin{aligned}
& \int_{a}^{b} u(x) \phi\left(\frac{1}{(\sigma(x)-a)} \int_{a}^{\sigma(x)} f(t) \Delta t\right) \frac{\Delta x}{x-a} \\
& +\int_{a}^{b} \int_{t}^{b} \phi\left(\left|f(t)-\frac{1}{(\sigma(x)-a)} \int_{a}^{\sigma(x)} f(t) \Delta t\right|\right) \frac{u(x)}{(\sigma(x)-a)(x-a)} \Delta x \Delta t \\
& \leq \int_{a}^{b} v(x) \phi(f(x)) \frac{\Delta x}{x-a},
\end{aligned}
$$

which coincides with inequality (2.3). Furthermore, the inequality sign in (3.3) is reversed if $\phi$ is subquadratic. 
Remark 3.3. The problem to characterize Hardy type inequalities with general weights and with general kernel is still an open question (see e.g. the books $[11,12,13]$ and the references there). However, when we have a special relation between the weights (see (3.3) and (3.2)), we can even have general kernel and refined Hardy inequality even for the case with time scales. In particular, by applying these inequalities with $\phi(u)=u^{p}$, which is superquadratic for $p \geq 2$ and subquadratic for $1<p \leq 2$, we obtain some new refined Hardy-type inequalities on time scales with "breaking point" $p=2$, which reduces to a new identity for $p=2$.

Our next aim is to present our result in a more general multidimensional setting. In the sequel we let $n \in \mathbb{Z}_{+}$and set $\mathbf{x}=\left(x_{1}, \cdots, x_{n}\right), \mathbf{t}=\left(t_{1}, \cdots, t_{n}\right) \in \mathbb{R}^{n}$, $\Omega_{1}, \Omega_{2} \subset \mathbb{R}^{n}$ are time scale measure spaces. Correspondinly, $[\mathbf{a}, \mathbf{b})$ means the set $\left[a_{1}, b_{1}\right) \times\left[a_{2}, b_{2}\right) \times \cdots \times\left[a_{n}, b_{n}\right), d \mathbf{x}=d x_{1} \cdots d x_{n}$ and $\mathbf{u}^{p}=\left(u_{1} \cdots u_{n}\right)^{p}$. Furthermore, for $\mathbf{x}, \mathbf{t} \in \mathbb{R}^{n}$ we write $\mathbf{x}<\mathbf{t}$ if componentwise $x_{i}<t_{i}, \quad i=1,2, \cdots, n$ and the relations $\leq, \geq$ and $<$ are defined analogously. Also for $\mathbf{a}, \mathbf{b} \in \mathbb{R}^{n}, \mathbf{a}<\mathbf{b}$, we define $(\mathbf{a}, \mathbf{b})=\left\{\mathbf{x} \in \mathbb{R}^{n}: \mathbf{a}<\mathbf{x}<\mathbf{b}\right\}$. Moreover, $(\mathbf{a}, \infty)=\left\{\mathbf{x} \in \mathbb{R}^{n}: \mathbf{a}<\mathbf{x}<\infty\right\}$ and the n-cells $[\mathbf{a}, \mathbf{b}),(\mathbf{a}, \mathbf{b}]$ and $[\mathbf{a}, \mathbf{b}]$ are defined similarly. In this setting, we obtain the following multidimensional time scale Hardy-type inequality with general kernel as follows:

Theorem 3.4. Let $\left(\Omega_{1}, \Sigma_{1}, \mu_{\Delta_{1}}\right)$ and $\left(\Omega_{2}, \Sigma_{1}, \mu_{\Delta_{2}}\right)$ be two time scale measure spaces with positive $\sigma-$ finite measures and let $u: \Omega_{1} \rightarrow \mathbb{R}$ and $k: \Omega_{1} \times \Omega_{2} \rightarrow \mathbb{R}$ be nonnegative such that $k(\mathbf{x},$.$) is a \mu_{\Delta_{2}}$-integrable function for $x \in \Omega_{1}$. Furthermore, suppose that $K: \Omega_{1} \rightarrow \mathbb{R}$ is defined by

$$
K(\mathbf{x})=\int_{\Omega_{2}} k(\mathbf{x}, \mathbf{y}) \Delta \mu_{2}\left(y_{1}\right) \cdots \Delta \mu_{2}\left(y_{n}\right)>\mathbf{0} . \quad \mathbf{x} \in \Omega_{1}
$$

and

$$
v(\mathbf{y})=\int_{\Omega_{2}} u(\mathbf{x}) \frac{k(\mathbf{x}, \mathbf{y})}{K(\mathbf{x})} \Delta \mu_{1}\left(x_{1}\right) \cdots \Delta \mu_{1}\left(x_{n}\right)<\infty, \quad \mathbf{y} \in \Omega_{2} .
$$

If $\phi:[\mathbf{a}, \infty) \rightarrow \mathbb{R}\left(a_{i} \geq 0, i=1, \cdots, n\right)$ is a nonnegative superquadratic function, then the inequality

$$
\begin{aligned}
& \int_{\Omega_{2}} u(\mathbf{x}) \phi\left(\left(A_{k} f\right)(\mathbf{x})\right) \Delta \mu_{1}\left(x_{1}\right) \cdots \Delta \mu_{1}\left(x_{n}\right) \\
& +\int_{\Omega_{2}} \int_{\Omega_{2}} u(\mathbf{x}) \frac{k(\mathbf{x}, \mathbf{y})}{K(\mathbf{x})} \phi\left(\left|f(\mathbf{y})-\left(A_{k} f\right)(\mathbf{x})\right|\right) \Delta \mu_{1}\left(x_{1}\right) \cdots \Delta \mu_{1}\left(x_{n}\right) \Delta \mu_{2}\left(y_{1}\right) \cdots \Delta \mu_{2}\left(y_{n}\right) \\
& \leq \int_{\Omega_{2}} v(\mathbf{x}) \phi(f(\mathbf{x})) \Delta \mu_{2}\left(x_{1}\right) \cdots \Delta \mu_{2}\left(x_{n}\right)
\end{aligned}
$$

holds for all nonnegative $\mu_{\Delta_{2}}$-integrable function $f: \Omega_{2} \rightarrow \mathbb{R}$ and for $A_{k} f:$ $\Omega_{1} \rightarrow \mathbb{R}$ defined by

$$
\left(A_{k} f\right)(\mathbf{x})=\frac{1}{K(\mathbf{x})} \int_{\Omega_{2}} k(\mathbf{x}, \mathbf{y}) f(\mathbf{y}) \Delta \mu_{2}\left(y_{1}\right) \cdots \Delta \mu_{2}\left(y_{n}\right), \quad \mathbf{x} \in \Omega_{1} .
$$

If $\phi$ is subquadratic, then the inequality sign in (3.4) is reversed. 
Proof. The proof is similar to to that of Theorem 3.1 so we omit the details.

Remark 3.5. For the one dimensional case $n=1$, Theorem 3.4 coincides with Theorem 3.1.

Corollary 3.6. Let $u: \Omega \rightarrow \mathbb{R}$ be nonnegative time scale measures and $k$ : $\Omega \times \Omega \rightarrow \mathbb{R}$ be nonnegative such that $k(\mathbf{x})$ is $\Delta$-integrable function. Furthermore, suppose that $K: \Omega \rightarrow \mathbb{R}$ is defined by

$$
K(\mathbf{x})=\int_{a_{1}}^{\sigma\left(x_{1}\right)} \cdots \int_{a_{n}}^{\sigma\left(x_{n}\right)} k(\mathbf{x}, \mathbf{t}) \Delta t_{1} \cdots \Delta t_{n}, \quad x_{i} \in\left[a_{i}, b_{i}\right)
$$

and

$$
v(\mathbf{t})=\int_{t_{1}}^{b_{1}} \cdots \int_{t_{n}}^{b_{n}} \frac{k(\mathbf{x}, \mathbf{t}) u(\mathbf{x})}{K(\mathbf{x})} \Delta t_{1} \cdots \Delta t_{n}, \quad t_{i} \in\left[a_{i}, b_{i}\right) .
$$

If $\phi: \Omega \rightarrow \mathbb{R}$ is a nonnegative superquadratic function, then the inequality

$$
\begin{aligned}
& \int_{a_{1}}^{b_{1}} \cdots \int_{a_{n}}^{b_{n}} \frac{k(\mathbf{x}, \mathbf{t}) u(\mathbf{x})}{K(\mathbf{x})} \phi\left(A_{k} f(\mathbf{x})\right) \Delta x_{1} \cdots \Delta x_{n} \\
& +\int_{a_{1}}^{b_{1}} \cdots \int_{a_{n}}^{b_{n}}\left(\int_{a_{1}}^{b_{1}} \cdots \int_{a_{n}}^{b_{n}} \frac{k(\mathbf{x}, \mathbf{t}) u(\mathbf{x})}{K(\mathbf{x})} \phi\left(\left|f(\mathbf{t})-A_{k} f(\mathbf{x})\right|\right) \Delta x_{1} \cdots \Delta x_{n}\right) \Delta t_{1} \cdots \Delta t_{n} \\
& \leq \int_{a_{1}}^{b_{1}} \cdots \int_{a_{n}}^{b_{n}} v(\mathbf{x}) \phi(f(\mathbf{x})) \Delta x_{1} \cdots \Delta x_{n}
\end{aligned}
$$

holds for all $\Delta$-integrable function $f: \Omega \rightarrow \mathbb{R}^{n}$ such that $f(\Omega) \subset \mathbb{R}^{n}$, where

$$
A_{k} f(\mathbf{x})=\frac{1}{K(\mathbf{x})} \int_{a_{1}}^{\sigma\left(x_{1}\right)} \cdots \int_{a_{n}}^{\sigma\left(x_{n}\right)} k(\mathbf{x}, \mathbf{t}) f(\mathbf{t}) \Delta t_{1} \cdots \Delta t_{n} .
$$

If $\phi$ instead is subquadratic, then the inequality sign in (3.5) is reversed.

Proof. This follows from Theorem 3.4 by setting $\Omega=\Omega_{1}=\Omega_{2}=\left[a_{1}, b_{1}\right)_{\mathbb{T}} \times$ $\left[a_{2}, b_{2}\right)_{\mathbb{T}} \times \cdots \times\left[a_{n}, b_{n}\right)_{\mathbb{T}} \subset \mathbb{R}^{n}, 0 \leq a_{i}<b_{i} \leq \infty$ for all $i \in\{i, \cdots, n\}, \Delta \mu_{1}(x)$ and $\Delta \mu_{1}(x)$ and $\Delta \mu_{2}(y)$ by the Lebesgue scale measures $\Delta \mathbf{x}$ and $\Delta \mathbf{t}$. Furthermore, replace $u(\mathbf{x}), v(\mathbf{t})$ and $k(\mathbf{x}, \mathbf{t})$ by $\frac{u(\mathbf{x})}{\mathbf{x}-\mathbf{a}}, \frac{v(\mathbf{t})}{\mathbf{t}-\mathbf{a}}$ and $k(\mathbf{x}, \mathbf{t})=\mathbf{1}$, if $0 \leq t_{i} \leq \sigma\left(x_{i}\right)$, $k(\mathbf{x}, \mathbf{t})=0$ if $t_{i}>\sigma\left(x_{i}\right)$, then the result follows and the proof is complete.

Remark 3.7. For the case $\mathbb{T}=\mathbb{R}$ and $n=1$ then Corollary 3.6 coincides with Theorem 2.3

Corollary 3.8. Let $u: \Omega \rightarrow \mathbb{R}$ be nonnegative time scale measures and $k$ : $\Omega \times \Omega \rightarrow \mathbb{R}$ be nonnegative such that $k(\mathbf{x})$ is $\Delta$-integrable function. Furthermore, suppose that $K: \Omega \rightarrow \mathbb{R}$ is defined by

$$
K(\mathbf{x})=\int_{a_{1}}^{\sigma\left(x_{1}\right)} \cdots \int_{a_{n}}^{\sigma\left(x_{n}\right)} \Delta t_{1} \cdots \Delta t_{n}=\prod_{i=1}^{n}\left(\sigma\left(x_{i}-a_{i}\right), \quad x_{i} \in\left[a_{i}, b_{i}\right)\right.
$$

and

$$
v(\mathbf{t}):=\int_{t_{1}}^{b_{1}} \cdots \int_{t_{n}}^{b_{n}} \frac{u(\mathbf{x})}{\prod_{i=1}^{n}\left(\sigma\left(x_{i}-a_{i}\right)\right.} \Delta x_{1} \cdots \Delta x_{n}, \quad t_{i} \in\left[a_{i}, b_{i}\right)
$$


If $\phi: \Omega \rightarrow \mathbb{R}$ is a nonnegative superquadratic function, then

$$
\begin{aligned}
& \int_{a_{1}}^{b_{1}} \cdots \int_{a_{n}}^{b_{n}} u(\mathbf{x}) \phi\left(\left(A_{k} f\right)(\mathbf{x})\right) \Delta x_{1} \cdots \Delta x_{n} \\
& +\int_{a_{1}}^{b_{1}} \cdots \int_{a_{n}}^{b_{n}}\left(\int_{a_{1}}^{b_{1}} \cdots \int_{a_{n}}^{b_{n}} u(\mathbf{x}) \phi\left(\left|f(\mathbf{t})-\left(A_{k} f\right)(\mathbf{x})\right|\right) \Delta x_{1} \cdots \Delta x_{n}\right) \Delta t_{1} \cdots \Delta t_{n} \\
& \leq \int_{a_{1}}^{b_{1}} \cdots \int_{a_{n}}^{b_{n}} v(\mathbf{x}) \phi(f(\mathbf{x})) \Delta x_{1} \cdots \Delta x_{n}
\end{aligned}
$$

holds for all $\Delta$-integrable function $f: \Omega \rightarrow \mathbb{R}^{n}$ such that $f(\Omega) \subset \mathbb{R}^{n}$, where

$$
\left(A_{k} f\right)(\mathbf{x})=\frac{1}{\prod_{i=1}^{n}\left(\sigma\left(x_{i}\right)-a_{i}\right)} \int_{a_{1}}^{\sigma\left(x_{1}\right)} \cdots \int_{a_{n}}^{\sigma\left(x_{n}\right)} f(\mathbf{t}) \Delta t_{1} \cdots \Delta t_{n} .
$$

If $\phi$ instead is subquadratic, then the inequality sign in (3.7) is reversed.

Proof. This follows directly from Theorem 3.1 by setting $\Omega=\Omega_{1}=\Omega_{2}=$ $\left[a_{1}, b_{1}\right)_{\mathbb{T}} \times\left[a_{2}, b_{2}\right)_{\mathbb{T}} \times \cdots \times\left[a_{n}, b_{n}\right)_{\mathbb{T}} \subset \mathbb{R}^{n}, 0 \leq a_{i}<b_{i} \leq \infty$ for all $i \in\{i, \cdots, n\}$, where $\mathbb{T}_{\mathbb{T}}$ is a time scale.by replacing the time scale measures and also by replacing $\Delta \mu_{1}(x)$ and $\Delta \mu_{2}(y)$ by the Lebesgue scale measures $\Delta \mathbf{x}$ and $\Delta \mathbf{t}$. Furthermore, replace $u(\mathbf{x}), v(\mathbf{t})$ and $k(\mathbf{x}, \mathbf{t})$ by $\frac{u(\mathbf{x})}{\mathbf{x}-\mathbf{a}}, \frac{v(\mathbf{t})}{\mathbf{t}-\mathbf{a}}$ and $k(\mathbf{x}, \mathbf{t})=\mathbf{1}$, if $a_{i} \leq t_{i}<\sigma\left(x_{i}\right) \leq b_{i}$, $k(\mathbf{x}, \mathbf{t})=\mathbf{0}$ if 0 otherwise. Then the result follows and the proof is complete.

Example 3.9. In Theorem 3.8, set $a_{i}=0$ for all $i=1,2, . ., n$, and $u(\mathbf{x})=\frac{1}{x_{1} \cdots x_{n}}$, then inequality (3.7) reduces to

$$
\begin{aligned}
& \int_{0}^{b_{1}} \cdots \int_{0}^{b_{n}} \phi\left(\left(A_{k} f\right)(\mathbf{x})\right) \frac{\Delta x_{1} \cdots \Delta x_{n}}{x_{1} \cdots x_{n}} \\
& +\int_{0}^{b_{1}} \cdots \int_{0}^{b_{n}}\left(\int_{t_{1}}^{b_{1}} \cdots \int_{t_{1}}^{b_{n}} \phi\left(\left|f(\mathbf{t})-\left(A_{k} f\right)(\mathbf{x})\right|\right) \Delta x_{1} \cdots \Delta x_{n}\right) \frac{\Delta t_{1} \cdots \Delta t_{n}}{t_{1} \cdots t_{n}} \\
& \leq \int_{0}^{b_{1}} \cdots \int_{0}^{b_{n}} \prod_{i=1}^{n}\left(1-\frac{x_{i}}{b_{i}}\right) \phi(f(\mathbf{x})) \frac{\Delta x_{1} \cdots \Delta x_{n}}{x_{1} \cdots x_{n}}
\end{aligned}
$$

If $\phi$ instead is subquadratic, then the inequality sign in (3.8) is reversed.

Remark 3.10. In Example 3.9, set $\mathbb{T}=\mathbb{R}, n=1$, and $\phi(x)=x^{p}(p \geq 2)$, then (3.8) yields the following result

$$
\begin{aligned}
& \int_{0}^{b_{1}}\left(\frac{1}{x} \int_{0}^{x} f(t) d t\right)^{p} \frac{d x}{x} \\
& +\int_{0}^{b}\left(\int_{t}^{b}\left|f(\mathbf{t})-\frac{1}{x} \int_{0}^{x} f(t) d t\right| d x\right) \frac{d t}{t} \\
& \leq \int_{0}^{b}\left(1-\frac{x}{b}\right) f^{p}(x) \frac{d x}{x}
\end{aligned}
$$

The inequality sign in (3.9) is reveresed if $1<p \leq 2$. 
Remark 3.11. There are very few multidimensional Hardy-type inequalities in the literature (see e.g. [11, 12, 13] and the references there). By applying our result with the function $\phi(u)=u^{p}$ as in Remark 3.3 we obtain some new such general refined Hardy type inequalities for the case when the weights are related by (3.6).

Acknowledgement. The first author who is a Senior Associate of the Abdus Salam International Centre for Theoretical Physics, Trieste, Italy acknowledged with thanks the hospitality and excellent facilities provided by the Centre to carry out this research. We thank the referee for some generous suggestions which have improved the final version of this paper.

\section{REFERENCES}

1. S. Abramovich, G. Jameson and G. Sinnamon, Refining of Jensen's inequality, Bull. Math. Soc. Sci. Math. Roumanie (N.S.) 47 (95) (2004), no. 1-2, 3-14.

2. J. Barić, R. Bibi, M. Bohner and J. Pečarić, Time scales integral inequalities for superquadratic functions, J. Korean Math. Soc. 50 (2013), 465-477.

3. R. Bibi, M. Bohner, J. Pečarić and S. Varošanec, Minkowski and Beckenbach-Dresher inequalities and functionals on time scales, J. Math. Ineq. 7 (2013), no. 3, 299-312.

4. M. Bohner and A. Peterson, Dynamic Equations on Time Scales: An Introduction with Applications, Birkhäuser Boston, Massachusetts, 2001.

5. M. Bohner and A. Peterson (eds.), Advances in Dynamic Equations on Time Scales, Birkhäuser Boston, Massachusetts, 2003.

6. T. Donchev, A. Nosheen and J. Pečarić, Hardy-type inequalities on time scale via convexity in several variables, ISRN Math. Anal. 2013 (2013), Article ID903196, 9pp.

7. G.H. Hardy, Note on a theorem of Hilbert, Math. Z. 6 (1920), 314-317.

8. G.H. Hardy, Notes on some points in the integral calculus, LX. An inequality between integrals, Messenger of Math. 54 (1925), 150-156.

9. G.H. Hardy, J.E. Littlewood and G. Pólya, "Inequalities", Cambridge University Press, Cambridge, 1959.

10. A. Kufner, L. Maligranda and L.E. Persson, The prehistory of the Hardy Inequality, Amer. Math. Monthly 113 (2006), 715-732.

11. A. Kufner, L. Maligranda and L.E. Persson, The Hardy Inequality - About its History and Some Related Results, Vydavatelsky Servis Publishing House, Pilsen, 2007.

12. A. Kufner and L.E. Persson, Weighted Inequalities of Hardy Type, World Scientific Publishing Co., Inc., Singapore, 2003.

13. A. Meskhi, V. Kokilashvili and L.E. Persson, Weighted Norm Inequalities for Integral Transforms with Product Kernels, Nova Science Publishers, Inc., New York, 2010.

14. J.A. Oguntuase and L.E. Persson, Hardy type inequalities via convexity - the journey so far, Austral. J. Math. Anal. Appl. 7 (2011), no. 2, Art. 18 , 19pp.

15. J.A. Oguntuase and L.E. Persson, Refinement of Hardy's inequalities via superquadratic and subquadratic functions, J. Math. Anal. Appl. 339 (2008), 1305-1312.

16. J.A. Oguntuase, L.E. Persson, E.K. Essel and B.A. Popoola, Refined multidimensional Hardy-type inequalities via superquadracity, Banach J. Math. Anal. 2 (2008), no. 2, 129139.

17. U.M. Özkan and H. Yildirim, Hardy-Knopp type inequalities on time scales, Dynam. Systems Appl. 17 (3-4) (2008), 477-486.

18. U.M. Özkan and H. Yildirim, Time scale Hardy-Knopp type integral inequalities, Commun. Math. Anal. 6 (1) (2009), 36-41. 
19. P. Řehák, Hardy inequality on time scales and its applications to half-linear dynamic equations, J. Inequal. Appl. 5 (2005), 495-507.

1 Department of Mathematics, Federal University of Agriculture, P.M.B. 2240, Abeokuta, Ogun State, Nigeria.

E-mail address: oguntuase@yahoo.com; oguntuaseja@unaab.edu.ng

2 Department of Mathematics, Lule̊̊ University of Technology, SE-971 87 LuleÅ, Sweden; Narvik University College, P.O. Box 385, N-8505 Narvik, Norway.

E-mail address: larserik@ltu.se 